\title{
Effects of ACE Inhibitors and Angiotensin Receptor Blockers in Normotensive Patients with Diabetic Kidney Disease
}

Authors

Dandan $\mathrm{He}^{1^{*}}$, Yaru Zhang ${ }^{1^{*}}$, Wei Zhang ${ }^{2}$, Yue Xing ${ }^{1}$, Yipeng Guo ${ }^{3}$, Fuzhen Wang ${ }^{4}$, Junya jia ${ }^{1}$, Tiekun Yan ${ }^{1}$, Youxia Liu ${ }^{1}$, Shan Lin ${ }^{1}$

Affiliations

1 Department of Nephrology, General Hospital of Tianjin Medical University, Tianjin, China

2 Department of Cardiac Surgery, Tianjin Chest Hospital, China

3 Department of Epidemiology, Tianjin Public Health Bureau, Tianjin, China

4 Department of Statistics, Fenyang Hospital of Shanxi Province, Fenyang, China

Key words

diabetic kidney disease, angiotensin-converting enzyme inhibitors, angiotensin receptor blockers, albuminuria, cardiovascular events, meta-analysis

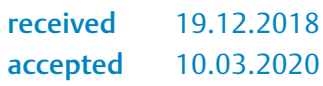

Shan Lin

Nephrology Department, Tianjin Medical University General Hospital

No. 154, Anshan Road

Heping District

Tianjin

China

Tel.: + 8622 60362531, Fax: + 862260362531

linshan1012@163.com $\circledast$ Supplementary Material for this article is available online at http://www.thieme-connect.de/products.

\section{ABSTRACT}

The role of angiotensin-converting enzyme inhibitors (ACEIs) and angiotensin receptor blockers (ARBs) in reducing the progression of albuminuria and risk of cardiovascular events in hypertensive patients with diabetic kidney disease (DKD) is well-documented. However, the efficacy and safety of these agents in normotensive patients with DKD are still controversial. MEDLINE, Embase, and Cochrane Library were searched for relevant random controlled trials. The odd risk (OR) reductions were calculated with a random-effects model. Decrease in albuminuria, changes in eGFR, major cardiovascular events, and drug-related adverse events were analyzed. Thirteen RCTs including 1282 patients were retrieved. Compared with placebo or other active agent groups, ACEls or ARBs significantly decreased albuminuria (MD $-80.28 \mathrm{mg} / \mathrm{d}, 95 \% \mathrm{Cl}-104.79 \mathrm{mg} / \mathrm{d}$ to $-55.77 \mathrm{mg} / \mathrm{d}$ ), and the efficacy is independent of changes in blood pressure and systolic blood pressure at baseline. The result of subanalysis showed the declining of albuminuria was more significantly in normotensive DKD patients with 2DM $(p=0.005)$. No significant differences were found with regard to the declining of evaluated glomerular filtration rate (eGFR) (MD $-0.29 \mathrm{ml} / \mathrm{min} / 1.73 \mathrm{~m}^{2}, 95 \% \mathrm{Cl}-2.99$ to $2.41 \mathrm{ml} / \mathrm{min} /$ $\left.1.73 \mathrm{~m}^{2}\right)$. There were no significant differences in the side effect of the drugs such as hypotension and hyperkalemia. This meta-analysis demonstrated that ACEls or ARBs can decrease albuminuria to varying degree in normotensive patients with $\mathrm{DKD}$, and better response occurred in patients with 2DM. 


\section{Introduction}

Diabetic kidney disease (DKD) occurs in almost $25-40 \%$ of patients with diabetes mellitus (DM) within 20-25 years of the onset of disease, and is responsible for end-stage kidney disease (ESKD) [1, 2]. Most people with DKD have hypertension and the blood pressure rises as albuminuria increases, however, some studies have shown that the incidence of microalbuminuria among type 2 diabetic patients without hypertension was approximately $40 \%$ [3]. And the increased blood pressure and microalbuminuria in normotensive patients with type 2 diabetes were associated with an increased cardiovascular risk [4].

Published guidelines recommend angiotensin-converting enzyme inhibitors (ACEls) or angiotensin II receptor blockers (ARBs) as the first line drugs for DKD patients with hypertension to reduce cardiovascular risk, kidney failure, and death [5]. Recent studies also provide evidence that treatment with ACEls or ARBs reduce the urinary albumin excretion rate (UAER) and retards the progression of kidney disease in normotensive diabetic patients [6, 7]. Some studies did not find the trend toward an antiproteinuric effect of ACEls or ARBs [8,9], which may be due to the short duration of observation or less of the patient included. This systematic review was therefore undertaken to assess the effects of ACEls and ARBs on kidney and cardiovascular outcomes in normotensive patients with DKD.

\section{Materials and Methods}

This systematic review of the literature was undertaken according to the approach recommended by the statement in PRISMA-P 2015. This meta-analysis included randomized controlled trials (RCTs) in which ACEls and ARBs was compared to placebo or an alternative antihypertensive agent in patients with normotensive diabetic kidney disease of various stages: microalbuminuria (albumin excretion $<30-300 \mathrm{mg} / \mathrm{d}$ ) or macroalbuminuria (albumin excretion $>300 \mathrm{mg} / \mathrm{d}$ ) with or without eGFR (estimated glomerular filtration rate) $60 \mathrm{ml} / \mathrm{min} / 1.73 \mathrm{~m}^{2}$.

\section{Search strategy and search selection}

Two authors independently searched from the following data sources without language restriction: MEDLINE via Ovid (from 1950 to August 2018), EMBASE (from 1966 to August 2018), and Cochrane Library databases using the MeSH headings and text words of all spellings of known ACE inhibitors and ARBs, RCTs, diabetic kidney disease, kidney outcomes and cardiovascular events.

We included all available RCTs, which compared ACEls/ARBs with placebo or other antihypertensive agents on the effects of kidney outcomes (including decrease in albuminuria, change of glomerular filtration rate), cardiovascular outcomes (defined as a composite of fatal or nonfatal myocardial infarction, angina, stroke, heart failure, and cardiovascular death), all-cause death, or drug-related adverse events [including hyperkalemia (commonly defined as serum potassium $>5.5 \mathrm{mmol} / \mathrm{l}$ ), cough, hypotension, allege, and edema] in patients with normotensive DKD. All completed RCTs that assessed the effects of ACEIs/ARBs compared with placebo or other antihypertensive drugs in normotensive patients with diabetic kidney disease, and which reported cardiovascular, renal or adverse outcomes, were eligible for inclusion. The exclusion crite- ria for the following studies are: (i) studies on population who suffered from DKD along with hypertension or other renal diseases. (ii) studies in patients undergoing dialysis or kidney transplantation. (iii) studies that fail to report the mean value or data necessary to estimate the standard deviation (SD) of the primary efficacy outcome.

\section{Data extraction and quality of evidence}

Two authors extracted data using standard data extraction forms, which included participants, interventions, comparisons, and outcomes. We used standard criteria (Jadad) to assess the quality of the trials (randomization, concealment of allocation, double blinding, withdraw and dropouts). Differences were resolved by consultation with a third reviewer.

\section{Statistical analysis}

We calculated odd risk (OR) and $95 \%$ confidence interval $(\mathrm{Cl})$ for each outcome by the random-effects model. For the continuous measurement of change of GFR, blood pressure and albuminuria, we used the weighted mean difference between groups. Regression analysis was conducted using the weighted mean difference of the systolic blood pressure at baseline and the weighted mean difference of the decrease of proteinuria to demonstrate that ACEIs/ARBs have the function of decreasing proteinuria independent of lowering blood pressure. Heterogeneity was analyzed beyond chance using the $I^{2}$ statistic to describe the percentage of variability. For data with high heterogeneity, sensitivity analysis was performed and based on the result we also performed a subanalysis in which participants were grouped by type 1 diabetes (1DM) and type 2diabetes (2DM). A 2-sided p-value less than 0.05 was considered statistically significant, and all statistical analyses were performed using STATA, version 12.0 and Review Manager 5.1 software.

\section{Results}

Our original search yielded 5073 articles, 4779 citations were excluded based on titles and abstracts. After a thorough and careful review, 13 trials which contained 1282 patients were included in our meta-analysis ( $\triangleright$ Fig. 1).

Of the contained 13 trials, 9 compared the efficacy of ACEls versus placebo, 1 compared ARBs with placebo, and 3 studies compared ACEls versus calcium channel blocker (CCB) and placebo. There are twelve studies included patients with eGFR greater than $60 \mathrm{ml} /$ $\mathrm{min} / 1.73 \mathrm{~m}^{2}$ [6-17] and eleven studies included patients with microalbuminuria [6-9,11-17]. Eight studies enrolled patients with type 1 DM $[6,8-11,13,15,18]$, four studies enrolled patients with type $2 \mathrm{DM}$ $[7,12,14,16]$ and one study included patients with type 1 and type 2 DM [17]. Follow-up ranged from 1 to 6 years. The characteristics of the included studies are presented in $>$ Table 1.

\section{Quantitative analysis}

Trial quality was variable. Allocation concealment was adequate in 7 trials $[6,7,12-16]$, inadequate in remaining 7 trials $[8-11,17,18]$. Nine studies were double-blind $[6-9,11,13,16-18]$, three were open $[10,14,15]$, and only one was single-blind [12]. Twelve $(85.7 \%)$ trials used an intention to treat analysis. The summary of the risk of bias is presented in $>$ Table 2 . 


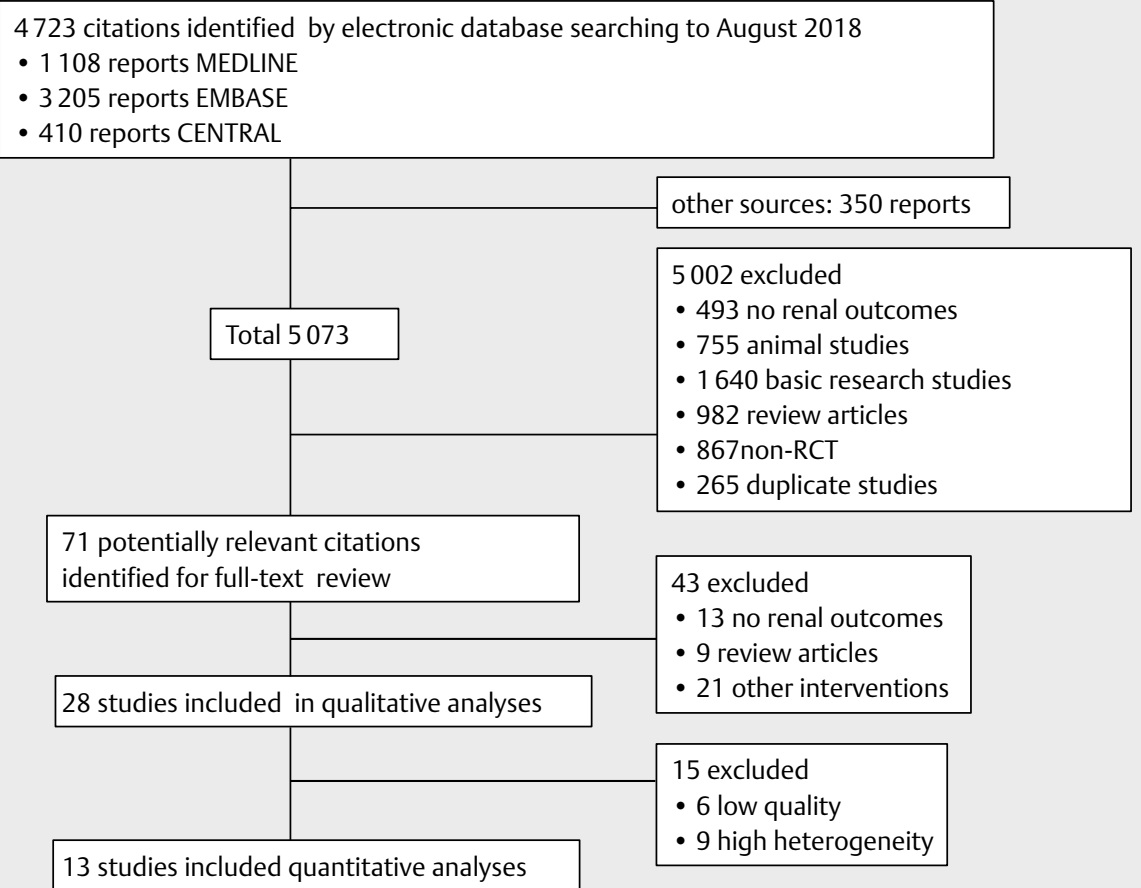

- Fig. 1 Process for identifying studies eligible for the meta-analysis.

\section{Decrease in albuminuria}

Data regarding the effects of ACEI/ARB on decrease in albuminuria were available from 7 trials $[1,6,7,12,13,17,18]$, including 5 trials ( $n=664$ ) of ACEl compared with placebo or active control therapy, and one trial $(n=163)$ of ARBs compared with placebo. The average decrease in albuminuria was $80.28 \mathrm{mg} / \mathrm{d}(95 \% \mathrm{Cl},-104.79 \mathrm{mg} / \mathrm{d}$ to $-55.77 \mathrm{mg} / \mathrm{d}$ ) less in patients receiving ACEIs/ARBs than in placebo or active control group patients $(p<0.001)$ and the heterogeneity analysis showed $\mathrm{I}^{2}=97 \%, \mathrm{p}<0.001$ ( $\triangleright$ Fig. 2 ).

According to the high heterogeneity, sensitivity analysis was conducted ( $\triangleright$ Fig. 1S), and the result showed that the heterogeneity was mainly caused by two studies $[7,18]$. Further study revealed the main difference between them was the types of diabetes in participants. The pathogenesis of type 1 and type $2 \mathrm{DM}$ is different, so we conducted a subanalysis according to type of diabetes in order to eliminate the effect of different types of diabetes on the reduction of albuminuria in the treatment of patients with ACEIs/ARBs. There were three studies reporting the decrease on albuminuria in patients with type 1 and type $2 \mathrm{DM}$, respectively. Data demonstrated the average decrease on albuminuria was $57.26 \mathrm{mg} / \mathrm{d}(95 \% \mathrm{Cl},-71.11 \mathrm{mg} / \mathrm{d}$ to $-43.40 \mathrm{mg} / \mathrm{d})$ and $99.82 \mathrm{mg} / \mathrm{d}(95 \% \mathrm{Cl},-125.72 \mathrm{mg} / \mathrm{d}$ to $-73.92 \mathrm{mg} / \mathrm{d}$ ), respectively in DKD patients with type 1DM and 2DM, less in patients receiving ACEIs/ARBs than in placebo or active control group patients. The results for subgroup differences showed that decrease on albuminuria in DKD patients with type 2DM was more significantly with ACEIs/ARBs ( $p=0.005, I^{2}=87.6 \%$; - Fig. 3).

\section{Relationship of albuminuria and blood pressure}

We also did analysis of the correlation between decreased levels of proteinuria and blood pressure, and found there was no significant association between two of them $(R=-0.23, p=0.55)$. And metaregression showed no association between decrease level of albuminuria and systolic blood pressure at baseline ( $p=0.323$; $>$ Fig. 4).

\section{Change of glomerular filtration rate}

Five trials comparing ACEls and placebo reported data on the change of glomerular filtration rate, and the results showed no statistically significant reduction in decline of GFR (MD $2.39 \mathrm{ml} / \mathrm{min} / 1.73 \mathrm{~m}^{2}$, $95 \% \mathrm{Cl}-1.29 \mathrm{ml} / \mathrm{min} / 1.73 \mathrm{~m}^{2}$ to $6.07 \mathrm{ml} / \mathrm{min} / 1.73 \mathrm{~m}^{2}$; $>$ Fig. 5) $[6,12,14,15,17]$ with no evidence of heterogeneity $\left(I^{2}=49 \%\right.$, $p=0.1)$. Only one study reported three cases of ESKD in 17 patients with placebo and zero in 15 patients with ACEls treatment [10], and the data showed no difference between two groups (OR 0.28, $95 \%$ Cl 0.00 to 1524.23 ). ( Fig. 2S, 3S)

\section{Cardiovascular disease outcomes}

Data in three studies $[6,7,13]$ including 302 patients reported 32 cardiovascular disease events. Of the 172 patients treated with ACEls there were 15 cardiovascular events (8.7\%) and 17 events occurred in 130 patients treated with placebo or active agents (13.1\%). Overall, ACEls and ARBs therapy did not reduce cardiovascular events versus placebo or other antihypertensive agents (OR $0.97,95 \% \mathrm{Cl} 0.45$ to 2.12 ) with no evidence of heterogeneity $\left(\mathrm{I}^{2}=0.0 \%, \mathrm{P}=0.95 ;\right.$ Fig. 6$)$.

\section{Total mortality}

Five studies reported 8 deaths in 335 patients with ACEls treatment (2.4\%) and 3 deaths in 301 patients with placebo or active agents therapy $(1.0 \%)[6,9-11,18]$. Overall, ACEI therapy did not reduce total mortality in patients with normotensive diabetic kidney disease 


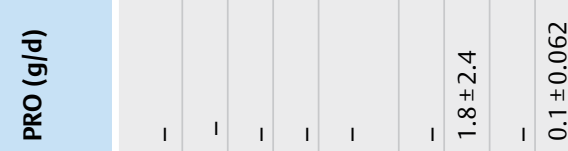

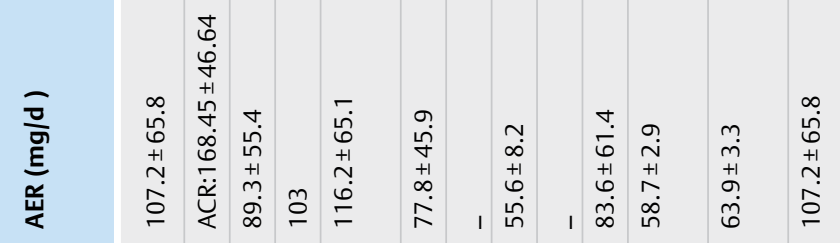

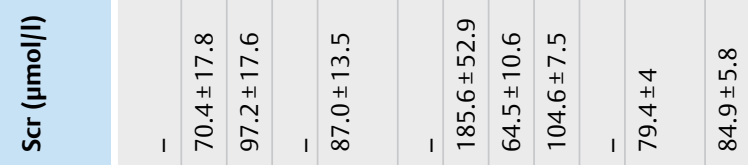

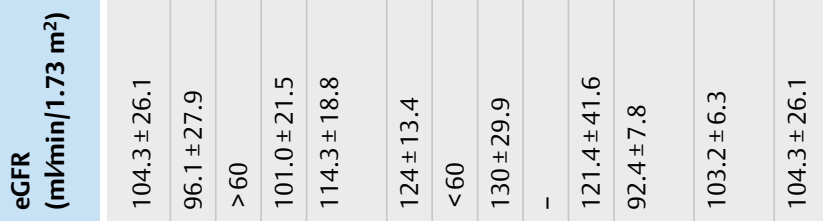

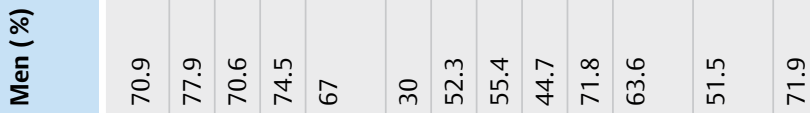

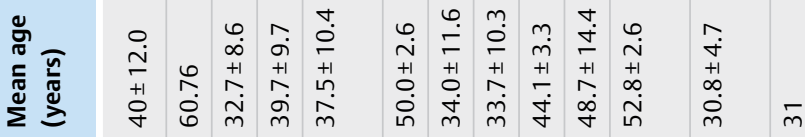

产焉竞

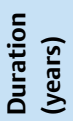

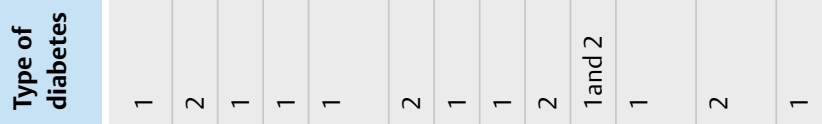

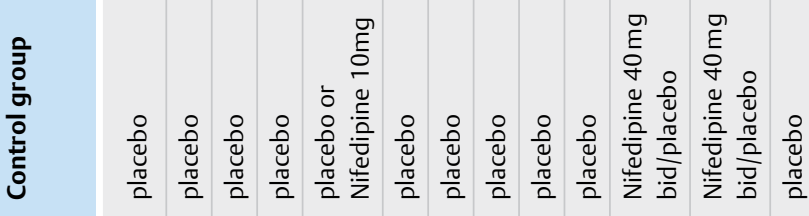

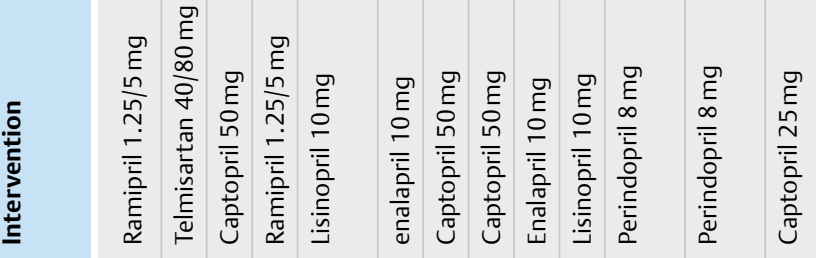

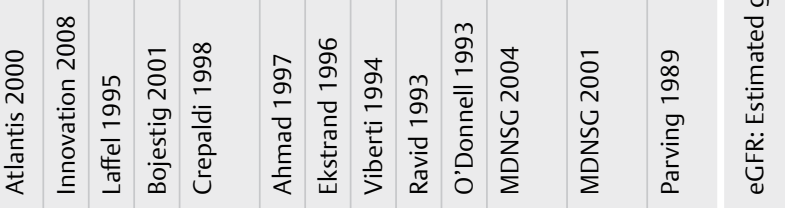


- Table 2 Quality assessment for included trials (Modified Jadad Score).

\begin{tabular}{|c|c|c|c|c|c|}
\hline Studies & Randomization & Concealment of allocation & Double blinding & Withdraws and dropouts & Total \\
\hline Atlantis 2000 & 2 & 1 & 1 & 1 & 5 \\
\hline Innovation 2008 & 1 & 0 & 2 & 1 & 4 \\
\hline Laffel 1995 & 1 & 0 & 1 & 1 & 3 \\
\hline Bojestig 2001 & 1 & 0 & 1 & 1 & 3 \\
\hline Crepald 1998 & 2 & 0 & 2 & 1 & 5 \\
\hline Ahmad 1997 & 1 & 1 & 2 & 1 & 5 \\
\hline Ekstrand 1996 & 1 & 0 & 2 & 1 & 4 \\
\hline Viberti 1994 & 1 & 0 & 2 & 1 & 4 \\
\hline Ravid 1993 & 2 & 0 & 2 & 0 & 4 \\
\hline O’Donnell 1993 & 1 & 0 & 2 & 1 & 4 \\
\hline MDNSG 2004 & 1 & 2 & 0 & 1 & 4 \\
\hline MDNSG 2001 & 1 & 2 & 0 & 1 & 4 \\
\hline Parving 1989 & 1 & 0 & 0 & 1 & 2 \\
\hline
\end{tabular}

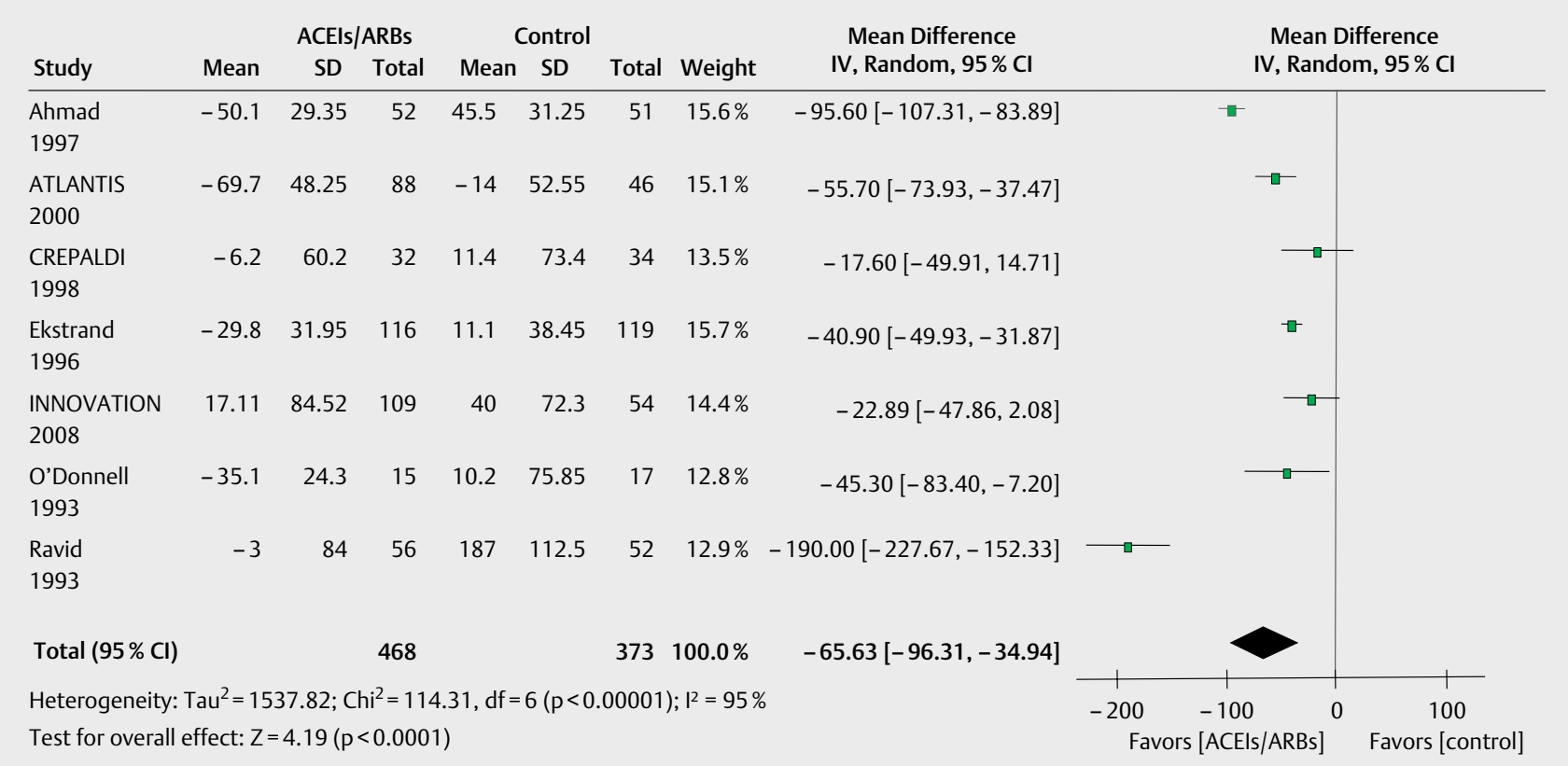

- Fig. 2 Effect of ACEls or ARBs compared with placebo or other active agents on albuminuria

(OR $1.09,0.16-7.20)$ with no evidence of heterogeneity $\left(I^{2}=0 \%\right.$, $\mathrm{p}=1.0$; Fig. 7).

\section{Adverse effects}

There were eight trials (931 patients) reported at least 1 adverse event [7-9, 11, 12, 16-18]. The data showed 33 adverse events occurred in 446 patients with ACEls treatment (7.4\%) and 34 events in 550 patients with placebo (7.2\%). Compared with control, ACEls/ ARBs therapy did not clearly increase the risk of adverse effects (OR 1.12, $95 \% \mathrm{Cl}$ 0.69-1.81; - Table 3). Among all kinds of adverse effects, cough is the most frequent occurrence, but the difference is not significant between two groups (OR 1.19, $95 \%$ Cl 0.66-2.12; - Table 3), the same as hypotension and other adverse effects.

\section{Risk of bias}

The Funnel plots and Begg's test applied to individual trials did not disclose any publication bias (Begg's Test: $\operatorname{Pr}>|z|=0.230$; $>$ Fig. 8).

\section{Discussion}

This meta-analysis including a total of thirteen studies with 1268 patients was conducted to investigate the efficacy and safety of 


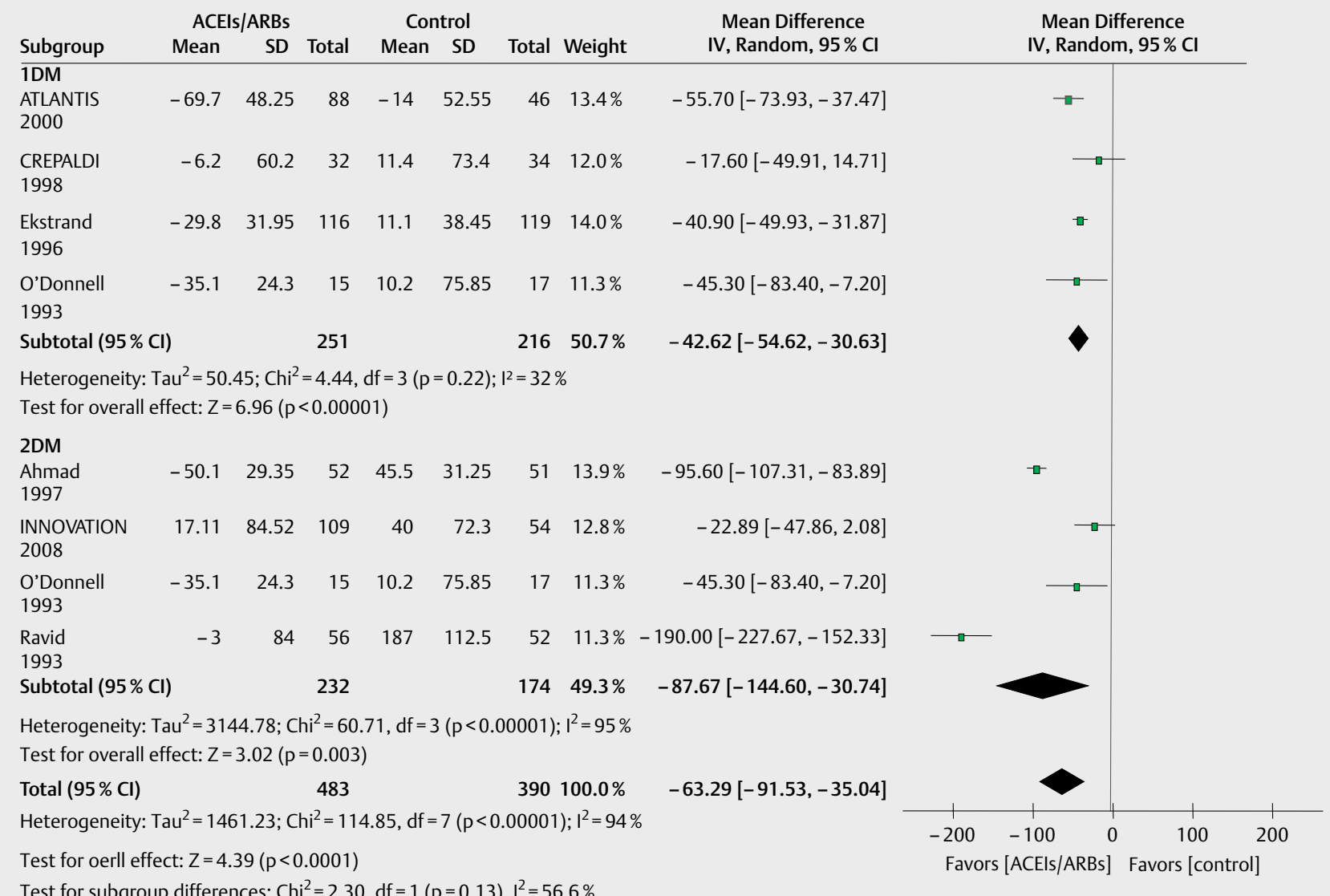

- Fig. 3 Sub-analysis for effect of ACEls or ARBs compared with placebo or other active agents on albuminuria.

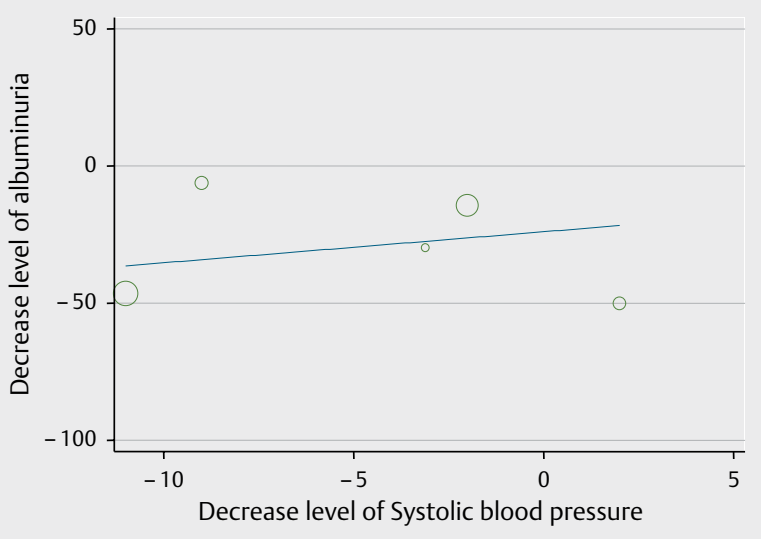

-Fig. 4 Meta-regression of decrease level of albuminuria on the decrease level systolic blood pressure

ACEIs/ARBs on renal and cardiovascular outcomes in normotensive patients with DKD. There was an obvious trend for a favorable effect for ACEIs/ARBs for decreasing of albuminuria, which was independent of the degree of blood pressure drop and systolic blood pressure at baseline. And the average level of decrease in albuminuria was more significantly in DKD patients with 2DM. However, no significant difference was observed on the risk of adverse effects. However, we did not find remarkable difference between the ACEIs/ ARBs and control groups regarding renal events, cardiovascular disease and total mortality.

There are several previously published meta-analyses and systematic reviews assessing the effects of RAAS blocking agents in patients with DKD, and found RAAS blocking agents are able to significantly reduce albuminuria. However, little data are available on the effect of early introduction of ACEIs/ARBs to normotensive patients with DKD. The initial reduction in albuminuria induced by ACEIs/ARBs was again shown in this study, which is consistent with previously published smaller trials of ACEIs/ARBs in normotensive patients with DKD $[6,7,12,13,16-18]$. We found ACEIs/ARBs therapy confers renal protective effects that are independent of changes in blood pressure in normotensive patients with DKD. And in the post-hoc analysis of the INNOVATION study [7], treatment with telmisartan not only prevents the progression of microalbuminuria, but also reverts from microalbuminuria to normoalbuminuria in Japanese normotensive type 2 diabetic patients. Early stages of diabetes mellitus are characterized by increases in intracapsular pressure and reduction in renal plasma flow despite the patients are normotensive [19]. Evidence from clinical trials and animal experiments suggests that the effect of renin angiotensin system (RAS) inhibitors in the kidney is to decrease efferent arteriolar resistance with resulting reduction of intraglomerular capillary pressure [10]. 


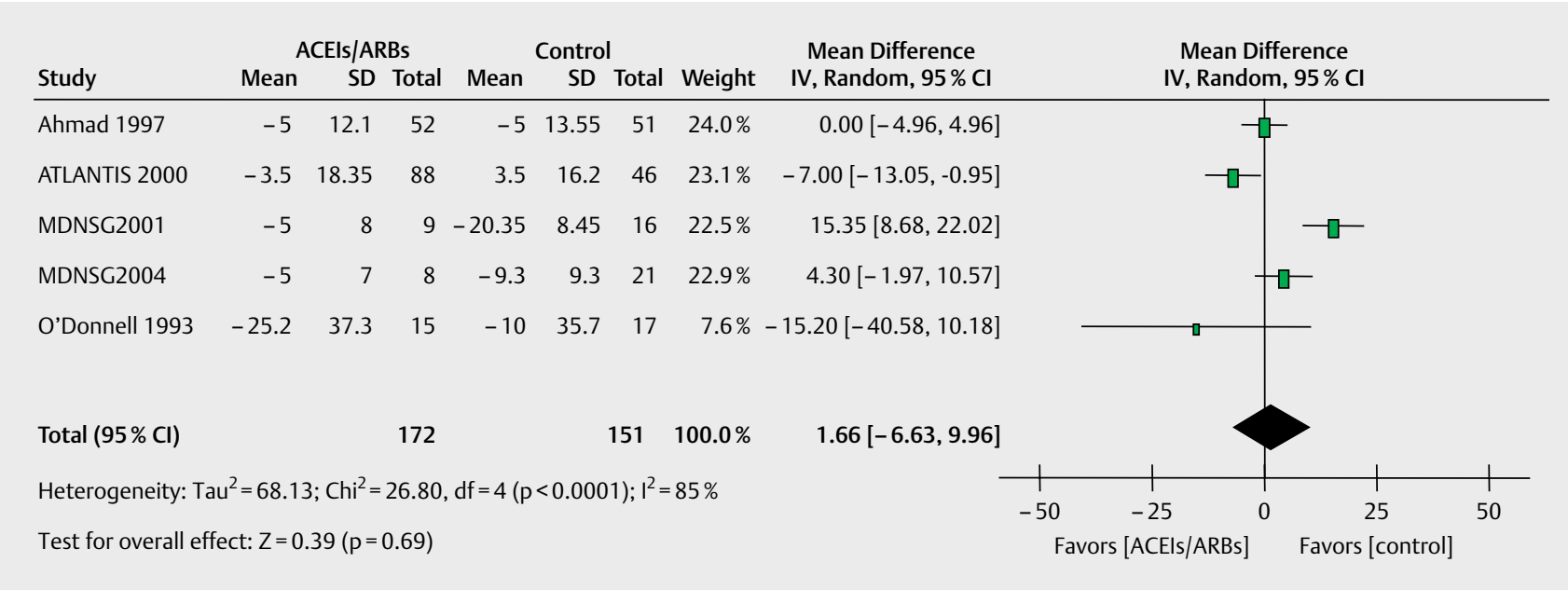

- Fig. 5 Effect of ACE-Is or ARBs compared with placebo on GFR.

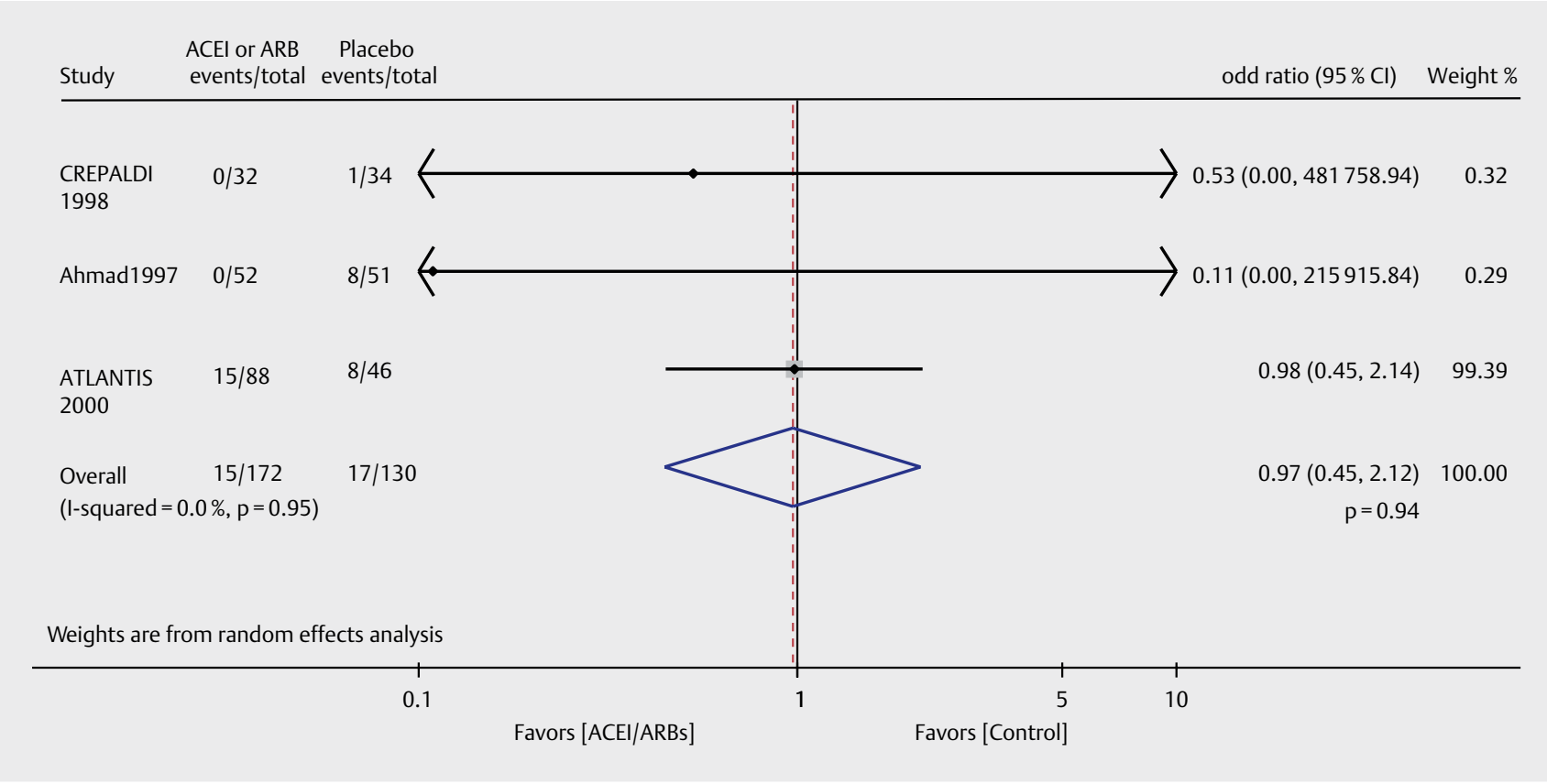

- Fig. 6 Effect of ACE-Is or ARBs compared with placebo or other active agents on cardiovascular disease outcomes.

In addition, increased vascular biomarkers of ACE indicate a vasomotor disturbance in this earlier stage of DKD (renal hyperfiltration). The glomerular hyperfiltration would be ameliorated by RAS inhibitors therapy $[20,21]$. These findings provide a likely explanation for the damaging effect of glomerular hypertension in the early stage of DKD and for the beneficial effect of ACEIs/ARBs even in patients without hypertension. We hypothesis that blood pressure drop may lead to glomerular hypoperfusion in patients with lower initial systolic blood pressure. In the present studies, adverse events were similar between two groups in terms of cough, hypotension and severe adverse [7-9, 11, 12,16-18] which suggest that ACEIs/ ARBs treatment in early stage of DKD is safe and well tolerated.

The development of microalbuminuria in diabetes mellitus strongly predicts ESRD and is associated with increased risk car- diovascular complications, as well as total mortality. Next, we analyzed endpoints of the rate of decline in GFR, ESRD, cardiovascular disease outcomes and total mortality, but found no significant difference between two groups. The relation between ACEIs/ARBs and later kidney events and cardiovascular disease outcomes in these patients has not been established, possibly because of the inclusion of only early-stage diabetic nephropathy and short follow-up periods. In all of contained studies, only one reported three cases of ESRD in placebo group and none in ACEls therapy [17]. The results demonstrate that $A C E I s$ could reduce the occurrence of ESRD, although the data showed no statistical significance. It is not yet clear whether ACEIs/ARBs can permanently prevent the deterioration of renal function. Further larger studies with longer follow-up times are required to elucidate the cardiovascular and renal 


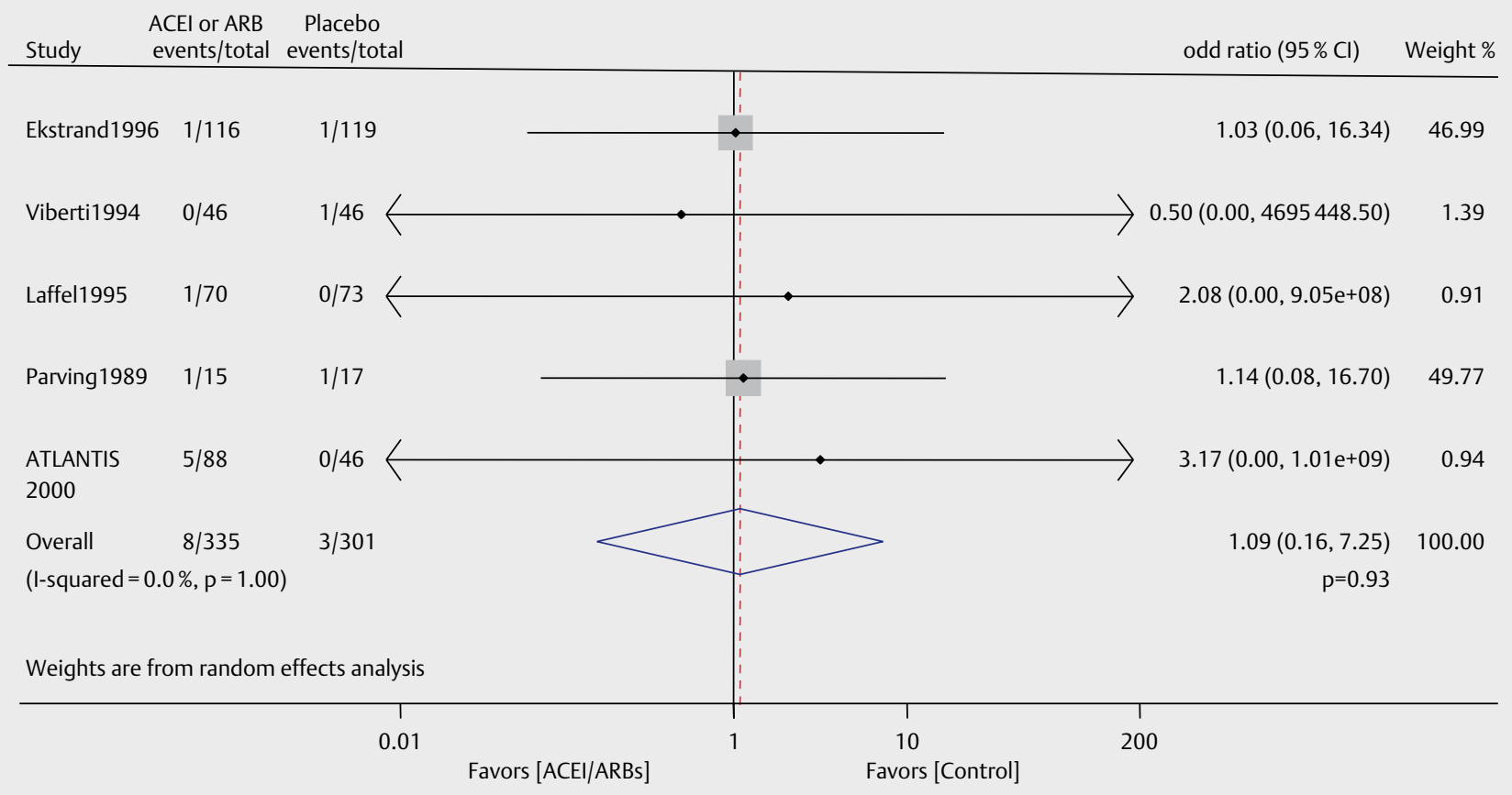

- Fig. 7 Effect of ACE-Is or ARBs compared with placebo or other active agents on total mortality.

- Table 3 Adverse events in the included RCTs.

\begin{tabular}{|l|l|l|l|l|}
\hline Adverse events & Studies reporting & ACEls/ARBs group n/n & Control group n/n & OR (95\%Cl) \\
\hline Total patients with adverse events & 8 & $33 / 446$ & $35 / 485$ & $1.12(0.69,1.81)$ \\
\hline Specific adverse events & & & & 0.64 \\
\hline Cough & 7 & $26 / 392$ & $21 / 376$ & $1.19(0.66,2.12)$ \\
\hline Hypotension & 1 & $1 / 70$ & $1 / 73$ & 0.56 \\
\hline Severe adverse & 1 & $14 / 109$ & $7 / 54$ & $0.99(0.42,2.31)$ \\
\hline OR: Odds ratio. & & & & 0.98 \\
\hline
\end{tabular}

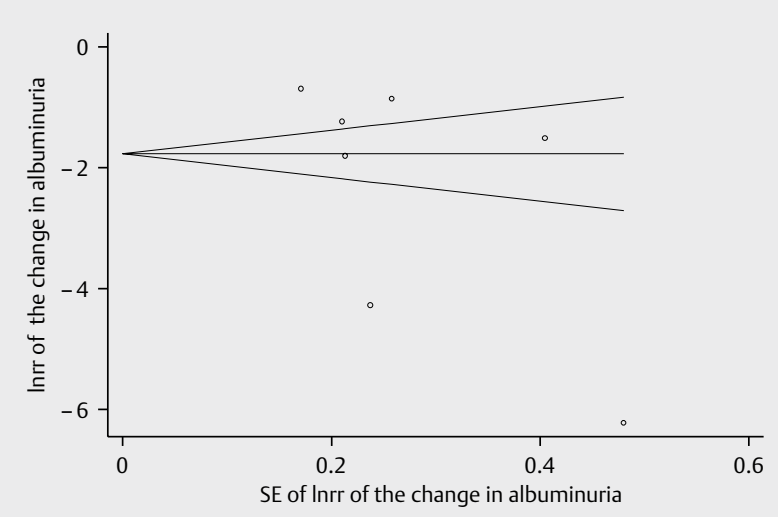

Fig. 8 Funnel plot of included studies in the analysis for effect of ACEls or ARBs compared with placebo or other active agents on albuminuria. protective effects of ACEIs/ARBs therapy in normotensive diabetic disease patients with incipient nephropathy.

There are several limitations of our meta-analysis that are inherent to the studies included. First, because of the lack of sufficient data, a subgroup analysis exploring the impact of ACEIs/ARBs on proteinuria is not conducted. Second, there is heterogeneity between different ACE inhibitors or ARBs, so different agents might not have the same risk-benefit ratio in DKD patients with normotension. Third, as most of the included RCTs were from developed western countries, there is a scarcity of data from other countries, which has limited the possibility to generalize the results.

\section{Conclusion}

This study demonstrated a reduction in albuminuria by RAAS blockade in normotensive patients with DKD, especially with 2DM, and side effects did not differ among the groups. More studies with 
longer follow-up times are required to elucidate the cardiovascular and kidney protective effects of ACEIs/ARBs therapy in these patients.

\section{Funding}

This work was supported by grants from the National Natural Science Foundation (81600553, 81270791, and 30800529), Tianjin Municipal Science and Technology Commission Foundation (14JCYBJC27900) and Tianjin Public Health Bureau Foundation (2014KR16), The 12th Five-Year Plan National Science and Technology Support Program (No. 2011BAI10B02) and Shanxi Public Health Bureau Foundation (201302051), the General Hospital of Tianjin Medical University Youth Incubation Foundation (ZYYFY2015001).

\section{Conflict of Interest}

The authors declare that they have no conflict of interest.

\section{References}

[1] Ritz E, Orth SR. Nephropathy in patients with type 2 diabetes mellitus. N Engl J Med 1999; 341: 1127-1133

[2] Zhang L, Long J, Jiang W et al. Trends in Chronic Kidney Disease in China. N Engl J Med 2016; 375: 905-906

[3] Basi S, Fesler P, Mimran A et al. Microalbuminuria in type 2 diabetes and hypertension: A marker, treatment target, or innocent bystander? Diabetes Care 2008; 31: (Suppl 2) S194-S201

[4] Marfella R, Esposito K, Giugliano D. Increase in nocturnal blood pressure and progression to microalbuminuria in diabetes. $N$ Engl J Med 2003; 348: 260-264 author reply 260-264

[5] Becker G], Wheeler D, De Zeeuw D et al. Kidney Disease: Improving Global Outcomes KDIGO clinical practice guideline for the management of blood pressure in chronic kidney disease. Kidney Int Suppl 2012; 37-414

[6] O’Hare P, Bilbous R, Mitchell T et al. Low-dose ramipril reduces microalbuminuria in type 1 diabetic patients without hypertension: Results of a randomized controlled trial. Diabetes Care 2000; 23: 1823-1829

[7] Makino H, Haneda M, Babazono T et al. Microalbuminuria reduction with telmisartan in normotensive and hypertensive Japanese patients with type 2 diabetes: A post-hoc analysis of The Incipient to Overt: Angiotensin II Blocker, Telmisartan, Investigation on Type 2 Diabetic Nephropathy (INNOVATION) study. Hypertens Res 2008; 31: 657-664
[8] Bojestig M, Karlberg BE, Lindström T et al. Reduction of ACE activity is insufficient to decrease microalbuminuria in normotensive patients with type 1 diabetes. Diabetes Care 2001; 24: 919-924

[9] Laffel LM, Mcgill JB, Gans DJ. The beneficial effect of angiotensin-converting enzyme inhibition with captopril on diabetic nephropathy in normotensive IDDM patients with microalbuminuria. North American Microalbuminuria Study Group. Am J Med 1995; 99: 497-504

[10] Parving HH, Hommel E, Damkjaer Nielsen M et al. Effect of captopril on blood pressure and kidney function in normotensive insulin dependent diabetics with nephropathy. BMJ 1989; 299: 533-6

[11] Viberti G, Mogensen CE, Groop LC et al. Effect of captopril on progression to clinical proteinuria in patients with insulin-dependent diabetes mellitus and microalbuminuria. European Microalbuminuria Captopril Study Group. JAMA 1994; 271: 275-279

[12] Ahmad J, Siddiqui MA, Ahmad H. Effective postponement of diabetic nephropathy with enalapril in normotensive type 2 diabetic patients with microalbuminuria. Diabetes Care 1997; 20: 1576-1581

[13] Crepaldi G, Carta Q, Deferrari G et al. Effects of lisinopril and nifedipine on the progression to overt albuminuria in IDDM patients with incipient nephropathy and normal blood pressure. The Italian Microalbuminuria Study Group in IDDM. Diabetes Care 1998; 21: $104-110$

[14] Jerums G, Allen T], Campbell D] et al. Long-term comparison between perindopril and nifedipine in normotensive patients with type 1 diabetes and microalbuminuria. Am J Kidney Dis 2001; 37: 890-899

[15] Jerums G, Allen TJ, Campbell D] et al. Long-term renoprotection by perindopril or nifedipine in non-hypertensive patients with type 2 diabetes and microalbuminuria. Diabet Med 2004; 21: 1192-1199

[16] Ravid M, Savin H, Jutrin I et al. Long-term stabilizing effect of angiotensin-converting enzyme inhibition on plasma creatinine and on proteinuria in normotensive type II diabetic patients. Ann Intern Med 1993; 118: 577-581

[17] O'Donnell M], Rowe BR, Lawson N et al. Placebo-controlled trial of lisinopril in normotensive diabetic patients with incipient nephropathy. J Hum Hypertens 1993; 7: 327-332

[18] Diabetologia Captopril reduces the risk of nephropathy in IDDM patients with microalbuminuria. The Microalbuminuria Captopril Study Group. Diabetologia 1996; 39: 587-593

[19] Futrakul N, Chaisuriya P, Ratanabanangkoon K et al. Microvascular dysfunction in normotensive, normoalbuminuric, normo- or hyperfiltrate type 2 diabetes. Ren Fail 2013; 35: 1191-1192

[20] Ustündağ B, Canatan H, Cinkilinç $\mathrm{N}$ et al. Angiotensin converting enzyme (ACE) activity levels in insulin-independent diabetes mellitus and effect of ACE levels on diabetic patients with nephropathy. Cell Biochem Func 2000; 18: 23-28

[21] Lozano-Maneiro L, Puente-García A. Renin-angiotensin-aldosterone system blockade in diabetic nephropathy. Present Evidences. J Clin Med 2015; 4: 1908-1937 